\title{
Mittelverteilung im schweizerischen Gesundheitswesen am Beispiel der Kantone Zürich und Basel-Stadt
}

\author{
D. Schopper ${ }^{a}$, R. Baumann-Hölzle ${ }^{b}$, F. Lange $^{b}$, I. Beeler ${ }^{c}$, F. Roth ${ }^{a}$, M. Tanner ${ }^{a}$
}

\section{Einführung}

In das schweizerische Gesundheitswesen flossen 1999 10,7\% des Bruttoinlandprodukts [2]. Im weltweiten Vergleich waren 1997 die Pro-KopfGesundheitsausgaben (in US-Dollar) 3724 \$ in den USA, 2644 \$ in der Schweiz, 2365 \$ in Deutschland und 2125 \$ in Frankreich [3]. Seitdem das neue Krankenversicherungsgesetz (KVG) 1996 in Kraft getreten ist, sind zudem die Krankenversicherungsprämien kontinuierlich und weit mehr als die Inflationsrate gestiegen. So entstand in den letzten Jahren eine Debatte über Mittelverteilung in der medizinischen Versorgung und über die Notwendigkeit, Leistungen im schweizerischen Gesundheitswesen systematisch zu beschränken, also zu rationieren [4-8]. Obwohl Uneinigkeit über das Ausmass der Rationierung herrscht, scheint die Notwendigkeit, dieses Thema öffentlich zu diskutieren und bessere faktische Grundlagen zu schaffen, allgemein akzeptiert zu sein. Im Kontext dieser aktuellen Debatte über Mittelverteilung in der medizinischen Versorgung beschlossen das Institut Dialog Ethik und das Schweizerische Tropeninstitut verschiedene Aspekte dieser Problematik beispielhaft in den Kantonen Zürich und BaselStadt zu untersuchen. Diese beiden Kantone wurden unter anderem gewählt, da hier die öffentliche Debatte auch dank der zuständigen politischen Instanzen schon weiter fortgeschritten ist als in anderen Gegenden der Schweiz. Obwohl diese beiden Kantone recht unterschiedlich sind betreffs Grösse, Bevölkerungsstruktur, Land-Stadt-Gefälle und Gesundheitsausgaben, geht es primär nicht um einen differenzierenden Vergleich, sondern um eine erste, beispielhafte Analyse von Trends und Wahrnehmungen, die auch für andere Situationen im Schweizer Gesundheitswesen gültig sein könnten. Die vorliegende Studie hatte vor allem auch das Ziel, weitere Diskussionen und vertieftere Studien auf Kantons- und nationaler Ebene anzuregen.
Auf der einen Seite arbeitet Dialog Ethik an der Umsetzung des «Zürcher Manifestes» [1]; andererseits versucht das Schweizerische Tropeninstitut aufzuzeigen, wie Erfahrungen aus der Gesundheitsplanung in Gebieten mit enormer Mittelknappheit für unsere Kantone kapitalisiert werden können. Zudem wurden beide Organisationen gebeten, im Rahmen der Diskussionen der Gesundheitskommission des Grossen Rates Basel-Stadt zur Rationalisierung und Rationierung Stellung zu nehmen. Sie setzten sich an mehreren öffentlichen wie akademischen Veranstaltungen gemeinsam für eine kohärente und konsistente Bearbeitung der Rationalisierungs- und Rationierungsproblematik ein. Die Studie wurde von beiden Instituten finanziert.

Die Studie bestand aus vier Komponenten:

1. einer Analyse der Ausgabentrends und Finanzierungsmechanismen im ambulanten und stationären Bereich in den beiden Kantonen im letzten Jahrzehnt;

2. einer Expertenbefragung zu Ausmass und Art der empfundenen Rationierung, Rationalisierung und Überversorgung;

3. einer Analyse der Medienberichterstattung zum Thema Mittelknappheit und

4. einer Zusammenfassung der Erfahrung anderer Länder, Entscheide über Prioritätensetzung im Gesundheitswesen zu fällen.

In diesem Artikel werden nur die ersten beiden Teile der Studie präsentiert und kommentiert [9]. Der vollständige Bericht der Studie liegt gedruckt vor, ist bei den beteiligten Instituten erhältlich und auf dem Internet zugänglich (www.sti.ch oder www.dialog-ethik.ch). 


\section{Methoden}

\section{Ausgaben [10]}

Die Ausgabentrends und Finanzierungsmechanismen im ambulanten und stationären Bereich in den Kantonen Zürich und Basel-Stadt [11] von 1990 bis 1999 wurden aufgrund existierender und zugänglicher Daten analysiert. Die folgenden Fragen sollten damit angegangen werden:

- Welche Ausgaben in der medizinischen Versorgung sind im letzten Jahrzehnt gestiegen und welche sind gefallen?

- Hat sich eine relative Verschiebung von stationärer hin zu ambulanter Behandlung ergeben?

- Hat eine Verlagerung der Finanzierung über Steuern zur Finanzierung über Krankenkassenprämien stattgefunden?

- Wer trägt die wirtschaftliche Last der Ausgabensteigerung?

Hierzu wurden Daten des Bundesamts für Statistik [12], der Finanzstatistik der öffentlichen Haushalte [13], der Behandlungsfallstatistik der santésuisse [14], der Angaben des Bundesamt für Sozialversicherungen [15] und des Kenndatenbuchs für den Kanton Zürich verwendet. Im stationären Bereich wurden zudem in acht Spitälern [16] Angaben aus den Jahresberichten der Jahre 1990-1999, ergänzt durch spitaleigene Angaben, analysiert und die Resultate mit einigen Spitaldirektoren anhand eines strukturierten Fragebogens diskutiert. Um die inflationsbereinigte Entwicklung der Ausgaben im Verlaufe der untersuchten Jahre darzustellen, wurden alle Ausgaben mit dem gesamtschweizerischen Konsumentenpreisindex [17] auf das Jahr 1990 indexiert. Es muss allgemein bemerkt werden, dass die schwierige Zugänglichkeit umfassender Datensätze und die zum Teil mangelhafte Qualität der Grunddaten der Primärstatistiken sowie die teilweise diskontinuierliche Datenerhebung die Aufarbeitung des Zahlenmaterials auf kantonaler Ebene und vor allem die Vergleichbarkeit der Angaben sehr erschwerten. So wurden z.B. bei den Spitälern bewusst die in den publizierten und zugänglichen Statistiken dargestellten Ausgaben als Grundlage verwendet; dies geschah vor allem auch deshalb, weil es im Verlaufe der Vorbereitungen deutlich wurde, dass wir kaum $\mathrm{Zu}$ gang zu den verschiedenen Betriebsbuchhaltungen erhalten konnten, was eine vertiefte Analyse ermöglicht hätte. Die hier präsentierten Resultate sind deswegen immer nur als Trendanalysen $\mathrm{zu}$ verstehen.

\section{Expertengespräche}

Insgesamt wurden 29 Expertengesprächen auf der Grundlage von Tiefeninterviews in den beiden Kantonen Basel-Stadt (14) und Zürich (15) bei folgenden Gruppen von Schlüsselpersonen durchgeführt: Ärzte (8) und Pfleger (5) aus der stationären Akutversorgung, stationäre Langzeitpfleger (3), ambulante Ärzte (5) und Pfleger (4). Die Psychiatrie wurde bei der Auswahl besonders berücksichtigt. Zudem wurden in beiden Kantonen Repräsentanten der Patientenorganisationen (3) und der Vertrauensärzte der Krankenkassen (3) befragt. Inhaltlich wurden folgende Themen behandelt: die von den Befragten dargestellte Definition von Rationierung, Rationalisierung und Überversorgung verbunden mit konkreten Beispielen aus dem beruflichen Alltag; Kriterien und Prozesse der Entscheidungsfindung und die Vorstellungen zur Rolle der verschiedenen Akteure; Wahrnehmung und Bewertungen der Entwicklung in den letzten 5-10 Jahren und Zukunftsvision. Aufbau und Stichprobenumfang bei diesen Tiefeninterviews mit Schlüsselinformanten orientierte sich an den Grundlagen für qualitative Forschung in den Sozialwissenschaften [18]. Die Tiefeninterviews wurden nach einem standardisierten, durch Pilottests erstellten Leitfaden geführt, auf Tonband aufgenommen und transkribiert. Die qualitative Analyse wurde mit ATLAS/ti vorgenommen. Ein kurzer semi-quantitativer Fragebogen, der die qualitativen Tiefeninterviews begleitete, wurde mit SAS analysiert.

\section{Resultate}

\section{Ausgabentrends}

Die uns zugänglichen Daten zeigten deutlich, dass im Kanton Zürich die Ausgaben der Krankenkassen, des Kantons und der Gemeinden für die medizinische Versorgung pro Einwohner von 1991 bis 1999 um 17\% angestiegen sind. In den beiden Basler Kantonen lag die Steigerung inflationsbereinigt bei 10,4\%. Bemerkenswert war der sehr starke Anstieg der Ausgaben pro Einwohner für ambulante Behandlungen zwischen 1996 und 1999 [19]: um 52,4\% in ZH und 27,8\% in BS/BL. Dabei stiegen die Ausgaben für Medikamente (etwa $23 \%$ in beiden Kantonen) und für ambulante Behandlungen im Spital (30,8\% in $\mathrm{ZH}$ und $37,4 \%$ in $\mathrm{BS} / \mathrm{BL}$ ) am stärksten. Im stationären Sektor waren die Ausgaben pro Einwohner in beiden Kantonen von 1991 bis 1999 leicht rückläufig $(-4,3 \%$ in $\mathrm{ZH}$ und $-1,8 \%$ in 


\section{Abbildung 1}

Prozentuale Entwicklung des Betriebsaufwandes in acht Spitälern zwischen 1990 und 1999 (zugrundeliegende Kostendaten auf 1990 indexiert).

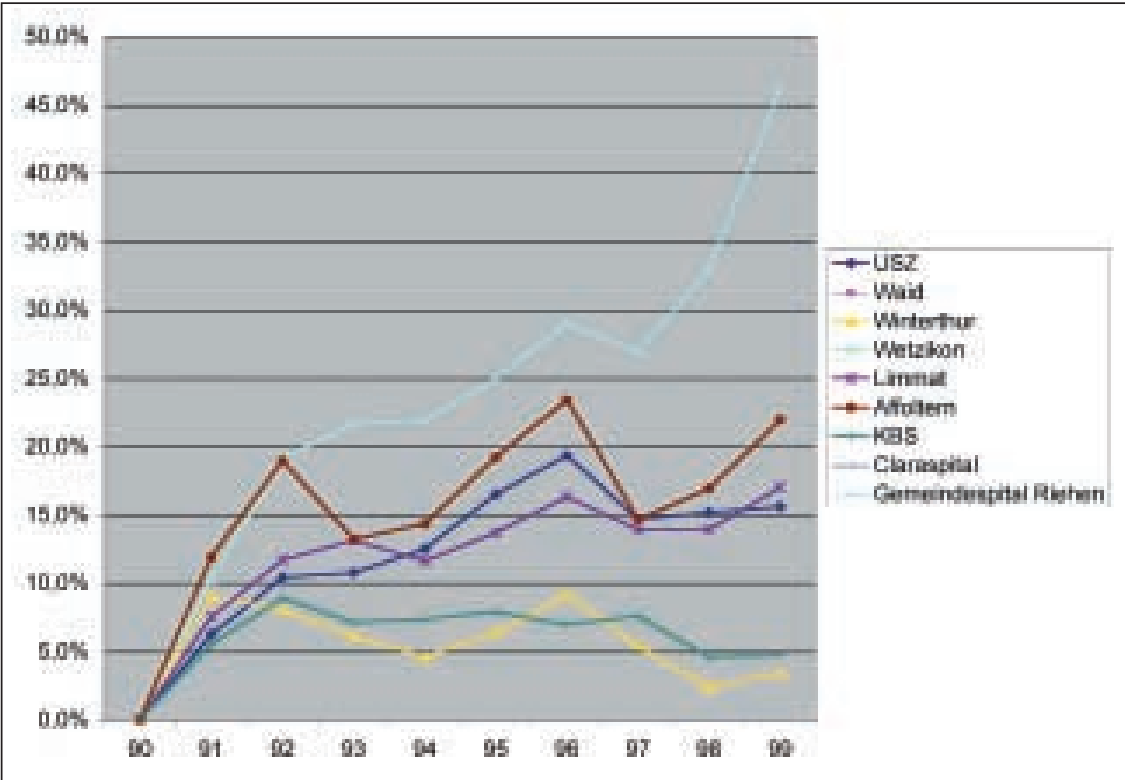

\section{Abbildung 2}

Prozentuale Entwicklung der Personalkosten und des Sachaufwandes im Universitätsspital Zürich (USZ) und im Kantonsspital Basel (KBS) zwischen 1990 und 1999 (zugrundeliegende Kostendaten auf 1990 indexiert).

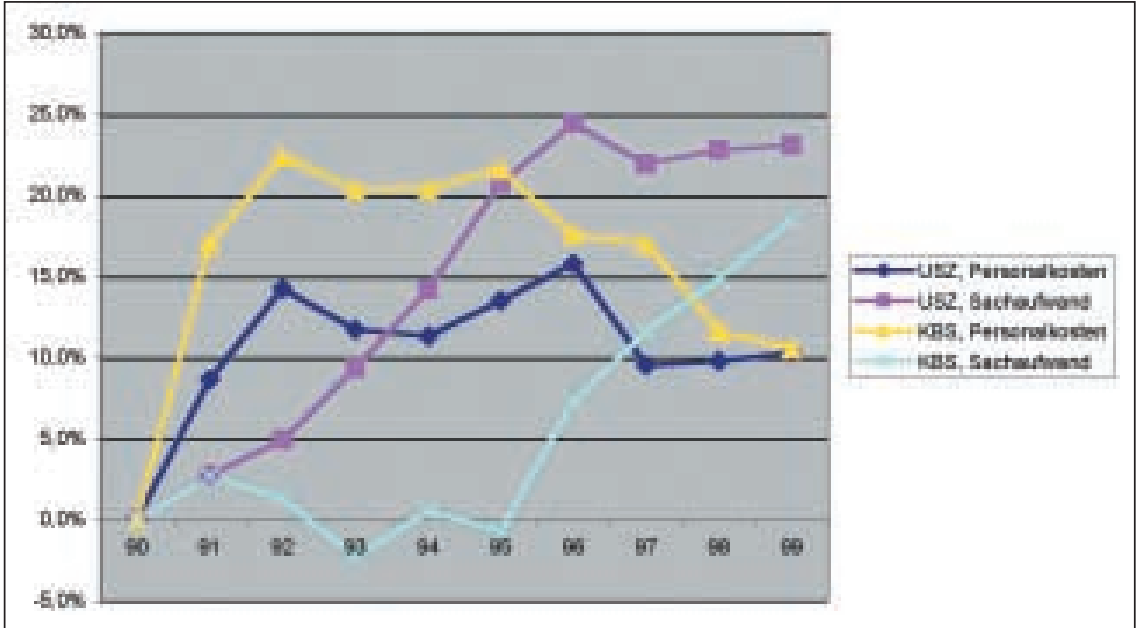

BS/BL). Die Stabilisierung und sogar Abnahme der Ausgaben im stationären Sektor hat Anfang bis Mitte der 90er Jahre stattgefunden. Seit 1997 sind die Ausgaben wieder steigend, aber ohne das Niveau von 1990 zu überschreiten. So ist der Ausgabenanstieg in den letzten zehn Jahren ausschliesslich auf den ambulanten Sektor zurückzuführen.

In scheinbarem Gegensatz zu dieser Ausgabenstabilisierung im stationären Sektor steht der Befund, dass in allen untersuchten Spitälern ausser dem Stadtspital Waid der Betriebsaufwand zunahm (Abb. 1). Nach Einführung des KVG 1996 verzeichnen die meisten Spitäler eine Senkung des Betriebsaufwandes, die jedoch ab 1998 wieder einer leichten Ausgabenzunahme weicht [20]. Leider konnten in dem von den Spitälern ausgewiesenen Betriebsaufwand die Ausgaben für ambulante und stationäre Leistungserbringung nicht getrennt untersucht werden. So muss angenommen werden, dass die Zunahme des Betriebsaufwandes v.a. auf den Ausbau der ambulanten Behandlungen zurückzuführen ist. Obschon die Angaben in den untersuchten Jahresberichten diesbezüglich lückenhaft sind und die Spitäler ambulante oder auch teilstationäre Behandlungen unterschiedlich ausweisen, ist dieser Befund aus folgenden Zahlen ersichtlich: Im Bezirksspital Affoltern stieg die Zahl der teilstationären Patienten zwischen 1990 und 1999 um 321,4\%, in Wetzikon die Zahl der teilstationären Behandlungen zwischen 1992 und 1999 um 262\% und die Taxpunktzahl aus teilstationären Behandlungen zwischen 1995 und 1999 um 276\%. Auch in Winterthur nahmen die Erträge aus teilstationärer Betreuung zwischen 1990 und 1999 um 102,5 \% zu und das Spital Limmattal richtete 1995 eine ambulante Chirurgie ein. Dieser Trend wurde uns auch eindeutig von seiten der befragten Spitaldirektoren bestätigt.

Der Spardruck, der von politischer Seite auf den stationären Sektor ausgeübt wurde, führte in den untersuchten Spitälern u.a. zu einer beträchtlichen Reduktion der Akutbetten (minus $10 \%-33,4 \%)$ und einer Kürzung der Aufenthaltsdauer in den Akutabteilungen (minus 14,2-31,7\%). Mit Ausnahme des Universitätsspitals Zürich (USZ) nahmen auch die Akutpflegetage ab, während die Patientenzahlen im gleichen Zeitraum stiegen. Interessant war festzustellen, dass nur eines der untersuchten Spitäler seit 1990 eine Statistik der Rehospitalisationen führte: In Wetzikon stieg die Zahl der Rehospitalisationen von 1990 bis 1996 um 376\%. Nach Angaben der Spitalberichte kam es im stationären Sektor hauptsächlich zu Einsparungen im Personalbereich und nicht zu einer Reduktion des medizinisch-technischen Angebotes, das offensichtlich ausgebaut und weiterentwickelt wurde. Abbildung 2 zeigt dies beispielhaft für die Universitätsspitäler beider Kantone. Innerhalb des Sachaufwandes nahm zudem der prozentuale Anteil des medizinischen Bedarfes [21] in allen untersuchten Spitälern stetig zu.

Seit Einführung des KVG fand in der gesamten Schweiz und besonders deutlich in den untersuchten Kantonen Zürich und Basel-Stadt/ -Landschaft eine ausgeprägte Verlagerung der Spi- 


\section{Abbildung 3}

Prozentuale Entwicklung des Betriebsertrages und der öffentlichen Unterstützung im Universitätsspital Zürich (USZ), im Kantonsspital Basel (KBS) und im Limmatspital in den Jahren 1990 und 1999 (zugrundeliegende Beträge auf 1990 indexiert).

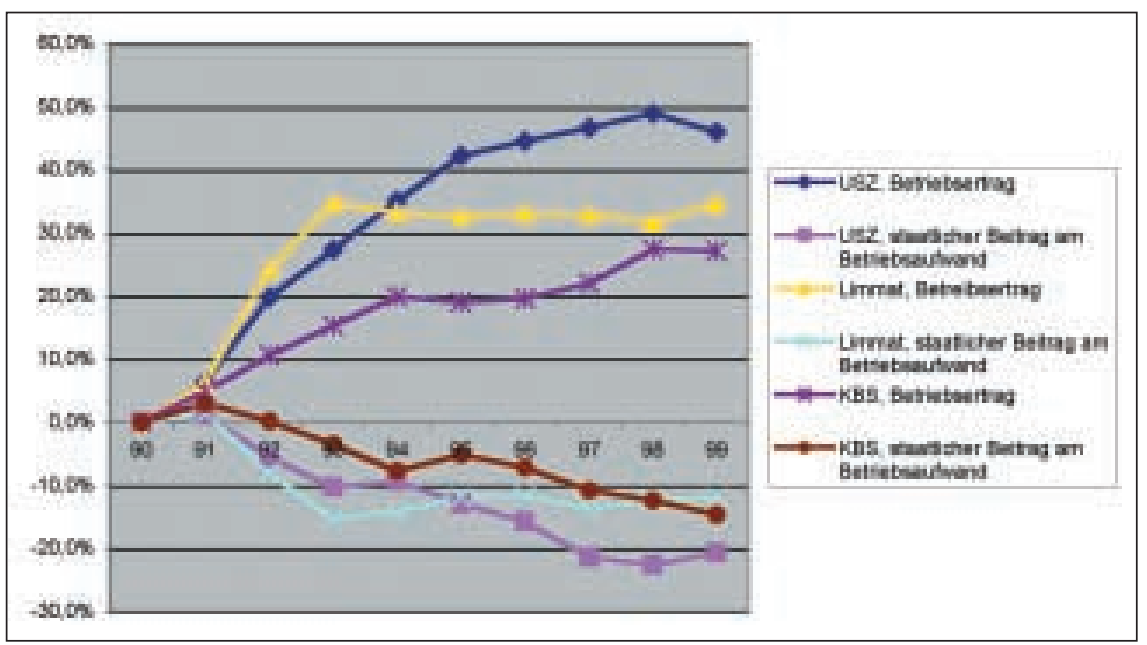

\section{Abbildung 4}

Entwicklung der Prämien und der Ausgaben der Krankenkassen (KK): Kanton ZH (pro Einwohner, indexiert auf 1990).

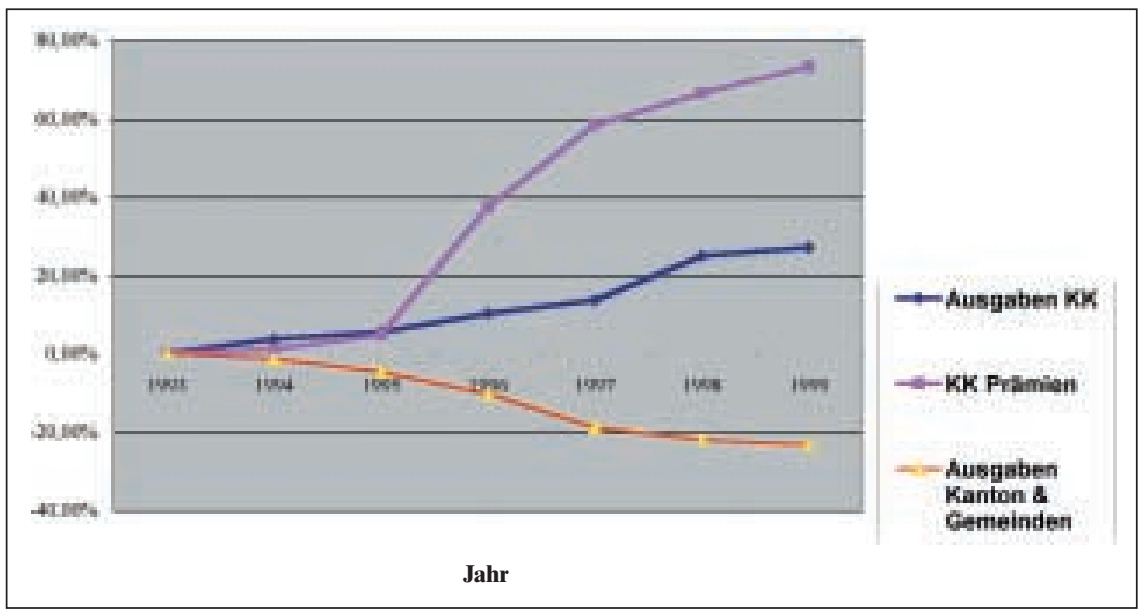

talfinanzierung über Steuergelder zur Finanzierung über Krankenkassenprämien statt. Der Anteil der staatlichen Beiträge am Betriebsaufwand war in allen betrachteten Spitälern rückläufig: am deutlichsten im USZ mit 20,8\%, am geringsten in Wetzikon mit 0,3\%. In Abbildung 3 ist exemplarisch an drei Spitälern die Entwicklung der Betriebserträge und staatlichen Beiträge (als Anteil am Betriebsaufwand) dargestellt. So bestätigte sich bei genauerer Betrachtung einiger öffentlicher Spitäler die allgemeine Beobachtung, dass die Spitalfinanzierung in den letzten Jahren in zunehmendem Masse über die Krankenkassen stattgefunden hat. Diese Verlagerung der Finanzierung von den Kantonen und Gemeinden hin zu den Krankenkassen manifestiert sich in der enormen Zunahme der Krankenkassenprämien. So erhöhten sich die Prämien in den beiden Basler Kantonen [22] von 1991 bis 1999 um real 92\%, bei einer gleichzeitigen Steigerung der Ausgaben der Krankenkassen, Kantone und Gemeinden für Krankenpflege von 10,3\%. Der Trend sah im Kanton Zürich ähnlich beunruhigend aus, wenn auch nicht ganz so ausgeprägt (Abb. 4). Diese steigende finanzielle Belastung, die insbesondere den Mittelstand und die unteren Einkommensschichten trifft, wird z.T. durch die Bundesund Kantonsbeiträge zur Prämienverbilligung abgefedert. Im Durchschnitt kamen 1998 31,4\% der Schweizer Bevölkerung in den Genuss dieser Verbilligungen [23]. Die Bezügerquote in den analysierten Kantonen war vergleichbar mit dem Schweizerischen Durchschnitt (ZH 29,8\%; BL $38,3 \%$; BS $27 \%)$.

\section{Rationierung und Überversorgung}

Die Expertengespräche, die die Empfindungen und Eindrücke von Schlüsselvertretern verschiedener Berufsgruppen erfassten, zeigten, dass in der Schweiz offenbar schon seit geraumer Zeit rationiert wird, wenn auch in viel geringerem Mass als in anderen industrialisierten Ländern [24]. «Harte» Rationierung, also jene Einschränkungen von notwendigen Leistungen, die mit eindeutig negativen gesundheitlichen Konsequenzen für den Patienten verbunden sind, wurde nur in acht von 35 Beispielen, also relativ selten, beschrieben (Abb. 5). Leistungseinschränkungen am Patienten waren abgelehnte Rehabilitationsund Spitalaufenthalte; die Notwendigkeit, Medikamente im Spital privat zu bezahlen; vorzeitige Spitalentlassungen; Situationen, in denen terminalen Patienten palliative Massnahmen vorenthalten wurden; reduzierte Pflegeleistungen; keine Überweisungen an Spezialärzte und Verweigerung von Leistungen, die nicht durch die Grundversicherung gedeckt werden. Diese Formen der Rationierung fanden fast ausschliesslich im stationären Bereich statt. Hingegen bezogen sich die beiden Beispiele zu den verweigerten Überweisungen an Spezialärzte auf HMO-Versicherte, also auf eine ambulante Situation mit Globalbudget. Überversorgung wurde hauptsächlich im stationären Sektor beschrieben und betraf u.a. die Aufenthaltsdauer in den Spitälern, Laboruntersuchungen, Diagnoseverfahren, die medikamentöse Versorgung, physiotherapeutische Leistungen, die Versorgung mit Herzschrittmachern und unnütze medizinische Behandlungen von terminalen Patienten.

Der wachsende ökonomische Druck und eine zunehmend bewusstere Mittelverteilung wurden hauptsächlich im stationären Bereich 


\section{Abbildung 5}

Klassifizierung der Rationierungsbeispiele (aufgrund der Tiefeninterviews mit Experten: Definition der «Rationierung» entspricht der subjektiven Wahrnehmung der befragten Experten).

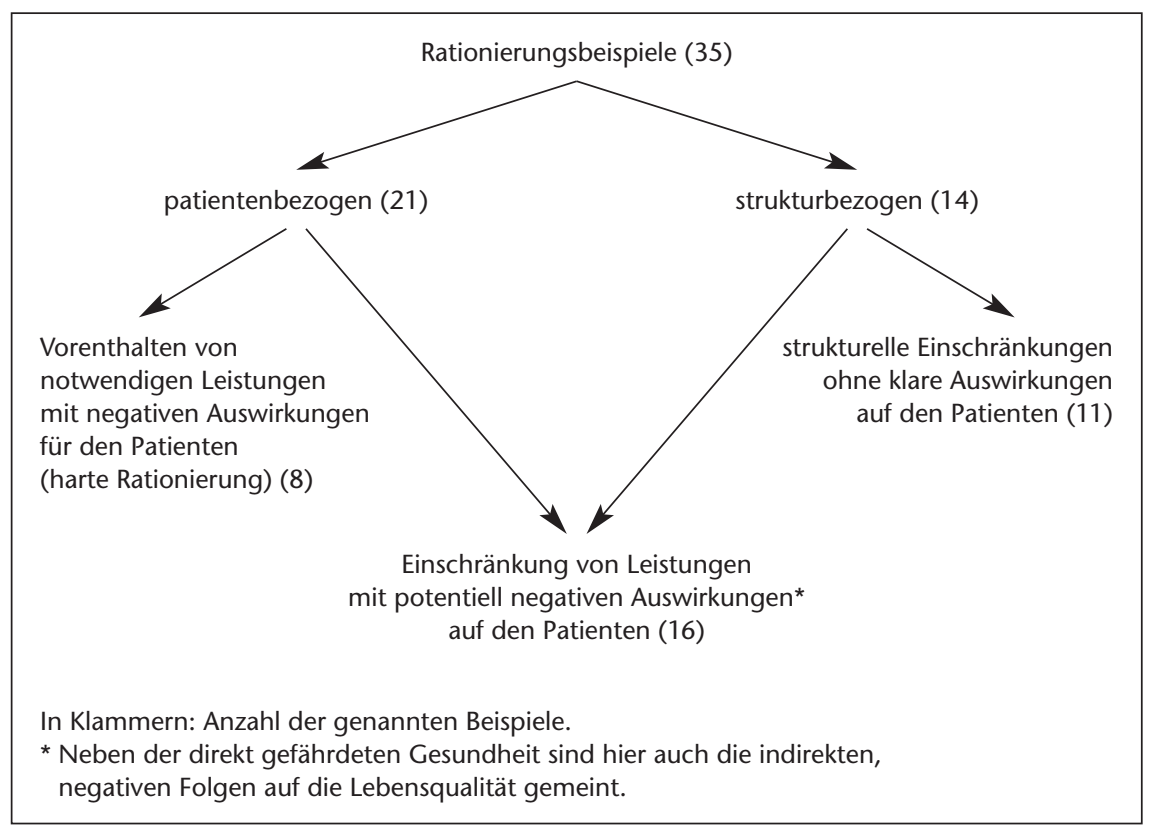

Neben der direkt gefährdeten Gesundheit sind hier
negativen Folgen auf die Lebensqualität gemeint.

vermerkt. Dabei wurde der rationellere Umgang mit den zur Verfügung stehenden Ressourcen positiv bewertet. Hingegen wurden viele Auswirkungen des Spardrucks als negativ empfunden; so z.B. verkürzte Spitalaufenthalte, gesteigerte Bettenauslastung, ständige Suche nach freien Betten, fehlendes Personal und ganz besonders die gesteigerten Arbeitsanforderungen. Die strukturellen Veränderungen, gepaart mit der Tatsache, dass stationäre Patienten vermehrt unter multiplen oder komplexeren Krankheitsbildern leiden, habe zu einer massiven Verdichtung der Arbeit geführt und würde den Patienten auf das zu behandelnde Krankheitssymptom reduzieren.

Entscheide über Mittelverteilung wurden auf der Makroebene von staatlichen Instanzen gefällt. Die Umsetzung dieser Entscheide trugen dann verschiedene Berufsgruppen im Gesundheitssektor. Im stationären Sektor wurde die Umsetzung auf der strukturellen Ebene oft in Führungsgremien gemeinsam diskutiert und beschlossen. Hingegen wurden sowohl im stationären als auch im ambulanten Sektor die Auswirkungen von Entscheiden auf der individuellen Ebene, d.h. den Patienten betreffend, dem behandelnden Arzt überlassen. In den acht Beispielen, die man als «harte» Rationierung bezeichnen kann, fehlten hinsichtlich frühzeitiger Spitalentlassungen und eingeschränkter Pflegeleistungen verbindliche Kriterien. Die Leistungserbringer wurden quasi in eine Art «Richterrolle» gezwungen und es war Zufall, welchen Patienten es traf. Patienten wurden im allgemeinen über den Rationierungsentscheid informiert, konnten ihn aber nicht beeinflussen. Es gab keine Instrumente oder Mechanismen, die eine aktive Rolle des Patienten vorsahen. In den ambulanten Rationierungsfällen wurde bemerkt, dass unter den spezifischen HMO-Arbeitsbedingungen, vor allem der Versorgung mit fixem Budget, Ärzte auch dazu neigen könnten, medizinische Kriterien der Ökonomie unterzuordnen. Innerhalb der verschiedenen Berufsgruppen zeigte sich viel Frustration über die Willkür der Entscheide «von oben» und die Schwierigkeit, diese am Patienten verantwortlich und kohärent umzusetzen. Es war nicht so sehr der Einzelfall, der unsere Interviewpartner beunruhigt, sondern vielmehr der erkennbare Trend hin zu unstrukturierten Rationierungsentscheiden. Die genannten Gründe für Beschränkungen der Mittelverteilung waren fast ausschliesslich ökonomischer Natur. Moralischethische Überlegungen schienen dabei bis jetzt kaum eine Rolle gespielt zu haben.

Die Meinungen über die Zukunft waren geteilt: die Hälfte der Experten hielt Rationierungen für notwendig bzw. sehr notwendig, die andere Hälfte hingegen bestritt die Notwendigkeit von Rationierungsmassnahmen, weil sich aufgrund des erwarteten wirtschaftlichen Aufschwungs die Finanzierungsspielräume wieder erweitern würden und weil im Bereich der Überkapazitäten noch ein grosses Einsparpotential vorhanden sei. Ausserdem wurden die fehlenden wissenschaftlichen Grundlagen für Rationierungsentscheide angeführt. Das Ausmass der medizinischen Überversorgung wurde von $3 / 4$ der Experten als hoch bis sehr hoch eingeschätzt. Aber obwohl der Abbau der Überversorgung von allen als notwendig angesehen wurde, fand sich in keiner Berufsgruppe eine Mehrheit, die meinte, dass in den nächsten 10 Jahren die Überversorgung reduziert würde. Für das Weiterbestehen oder die Zunahme der Überversorgung wurden gleichermassen Leistungserbringer und Patienten verantwortlich gemacht. Das Nebeneinander von Rationierung und Überversorgung im stationären Bereich führte in der Expertenbefragung zu einem bedeutenden Widerspruch. Es wurde die Meinung geäussert, wenn man nur genügend von den unnötigen Leistungen abbaue, sei Rationierung nicht notwendig. Gleichzeitig wurde aber angenommen, dass die Überkapazitäten bestehen bleiben oder sogar noch zunehmen würden. Dies erkläre sich dadurch, dass jegliche Reform der Mittelverteilung auf grosse Widerstände stosse, da Einzelinteressen im Vordergrund stünden und niemand die Verantwortung für Entscheidungen tragen möchte. 


\section{Diskussion}

Unsere Analyse in den Kantonen Zürich und Basel-Stadt belegt vielfach geäusserte Vermutungen der letzten Jahre: Die Ausgaben im Gesundheitswesen steigen weit mehr als die Inflationsrate und dies besonders im ambulanten Bereich. Betrachtet man die Ausgabenentwicklung in $\mathrm{Zu}$ sammenhang mit den Befunden der Expertenbefragung, ergeben sich einige interessante Einsichten, die durch weiterführende vergleichende Studien noch überprüft werden sollten:

1. Der Spardruck im stationären Sektor hat in den letzten 10 Jahren zu strukturellen Beschränkungen wie reduzierte Krankenhausbettenzahl, kürzere Aufenthaltsdauer für stationäre Patienten und Abbau des stationären Pflegepersonals geführt, die zum Teil als Rationierung wahrgenommen werden. Eine stark gestiegene Rehospitalisationsrate in dem einzigen Spital, das dies untersucht hat, und Fallbeispiele von negativen Auswirkungen auf den Gesundheitszustand des betroffenen Patienten deuten darauf hin, dass strukturelle Beschränkungen zum Vorenthalt von medizinisch sinnvollen Massnahmen führen können.

2. Eine Verschiebung der Ressourcen von der Pflege hin zur medizinischen Technologie konnte im stationären Sektor beobachtet werden. Dies geschah offenbar unter anderem, um bei gleichbleibendem Ausgabenniveau den steigenden Ansprüchen der Patienten (und Ärzte) an eine Spitzenmedizin gerecht zu werden. Die Ausgabenanalyse unterstützt damit die Befunde der Expertengespräche, wonach die Ressource «Menschlichkeit» sowohl auf Kosten der Patienten als auch auf Kosten des Personals im Gesundheitswesen rationiert wird, während gleichzeitig eine medizin-technische Überversorgung besteht. Verdeckte Rationierung erscheint damit als Antwort auf selektiven Spardruck im betroffenen stationären Sektor.

3. Gleichzeitig hat der beschriebene Spardruck zu einem Ausweichen auf den ambulanten Sektor geführt. Die höheren Ertragsmöglichkeiten bei ambulanten Behandlungen könnten für die Spitäler ein grosser Anreiz sein, damit die Betriebsergebnisses zu verbessern. Diese Verlagerung der Ausgaben kommt die Krankenkassen «teurer» zu stehen, was wiederum die steigenden Krankenkassenprämien erklären könnte. Obwohl der Bürger dies als steigende «Kosten» wahrnimmt, ist anzunehmen, dass die Verlagerung der medizinischen Versorgung vom stationären zum ambulanten Bereich in Wirklichkeit eine Kostensenkung bewirkte. Das allgemeine Credo, das international und auch in der Schweiz vertreten wird, sagt, dass dieselbe Behandlung ambulant vorgenommen weniger kostet als stationär. Gerade in diesem Bereich ergeben sich nun verschiedene Kernfragen, die vertiefte Studien verlangen.

Sinn dieser Studie war es, einen ersten Einblick in die Art und Auswirkung der Mittelverteilung in der medizinischen Versorgung zu geben, insbesondere im Blick auf das Problem der Rationierung. Wir wurden in unserem Anliegen auch mit der grossen Schwierigkeit konfrontiert, anhand der verfügbaren, lückenhaften und zu wenig detaillierten Daten zu untersuchen, wie Ausgabentrends und Finanzierungsmechanismen die Leistungserbringung in den verschiedenen Bereichen der medizinischen Versorgung (z.B. ambulant/stationär, Pflege/medizinisch-technische Leistungen) beeinflussen und wie sich das schliesslich auf die betroffenen Patienten auswirkt. So kann die hier zusammengefasste Untersuchung auch nur ein erster Versuch sein, diese Schnittstellen $\mathrm{zu}$ beleuchten und damit die nötigen und wichtigen Vertiefungsstudien anzuregen.

\section{Erste Schlussfolgerungen}

Die Ausgaben werden sowohl im stationären als auch im ambulanten Sektor weiterhin stark ansteigen. Das ist einerseits auf eine «Explosion» der Lohnkosten im stationären Sektor zurückzuführen, die über mehrere Jahre tief gehalten wurden, und andererseits auf eine anscheinend nicht aufzuhaltende Spirale des Angebots und der Nachfrage nach teuren Medikamenten und medizinisch-technischen Behandlungen. Die grosse Gefahr besteht darin, dass ohne ganzheitliches Konzept selektiver Spardruck in einem Bereich verdeckte Rationierungsmassnahmen bewirkt und gleichzeitig ein Ausweichen auf andere Bereiche fördert. Im Endeffekt entsteht dadurch eine neue Ausgabensteigerung, die wiederum zu Sparmassnahmen mit unerwünschten Konsequenzen führt. Dabei ist wichtig festzustellen, wie sehr die betroffenen Berufsgruppen bereits seit geraumer Zeit die Rationierung wahrnehmen, was dringend nach weiterführenden Studien zur objektiven Beurteilung der Rationierungslage verlangt.

Ein unkontrollierter oder gleitender Übergang in einschneidende Rationierungmassnahmen könnte vermieden werden, wenn im 
Schweizer Gesundheitswesen auf nationaler Ebene Prioritäten für die Mittelverwendung in der medizinischen Versorgung gesetzt würden. Dazu bedarf es einerseits einer gezielten Datenerhebung und -analyse, gepaart mit einer transparenten Informationspolitik, und andererseits einer breiteren gesellschaftlichen und politischen Diskussion zum Thema Mittelverteilung und Rationierung, deren Teilnehmer alle Betroffenen, d.h. Politiker, Leistungserbringer, Krankenkassen und Bürger, sein sollten. Diese Diskussion über eine faire Leistungs- und Mit- telverteilung im schweizerischen Gesundheitswesen bedarf eines expliziten politischen Auftrages und klarer Strukturen. In einem ersten Schritt sollten gemeinsam die Ziele festgelegt werden, auf welche die Diskussion überhaupt hinführen soll. Voraussetzung für diesen Meinungsbildungsprozess ist der politische Wille, Verantwortung für eine faire Leistungs- und Mittelverteilung zu übernehmen und Prioritäten zu setzen. Schweden geht hier zurzeit einen beispielhaften Weg, von dem die Schweiz vielleicht einiges lernen könnte (siehe [25]). 


\section{Literatur}

1 Sonderbeitrag: Rationierung im Gesundheitswesen. Schweiz Ärztezeitung 1999;80(45):2635-71.

2 Bundesamt für Statistik, 14 Gesundheit, Kosten des Gesundheitswesens, Pressemitteilung Nr. 035-0106-40, Neuchâtel Juli 2001.

3 World Health Report 2000. Health systems: improving performance. WHO, Geneva.

4 Müller AF. Sélection entre malades? Schweiz Ärztezeitung 1996;77(50):2034-5.

5 Brunner HH. Formen der Rationierung medizinischer Leistungen. Schweiz Ärztezeitung 1997;78(9):292-9.

6 Vassalli P. Le Rationnement des soins de santé. Pourquoi, comment, par qui? Documentation, Forum Louis-Jeantet sur les coûts de la santé 1998, Genève.

7 Bericht der Gesundheitskommission des Grossen Rates. Rationierung im Gesundheitswesen? 26. Januar 2000 / 06441, Basel-Stadt.

8 Sommer JH. Muddling through elegantly: Rationierung im Gesundheitswesen. Reihe Gesundheitsökonomie. Basel: EMH Schweizerischer Ärzteverlag; 2001.

9 Um die Lesbarkeit des Texts zu erhöhen, wird in diesem Artikel nur die männliche Form benutzt (z.B. Arzt, Patient, Bürger), die aber sowohl die männliche als auch die weibliche Person beinhaltet.

10 Es wird explizit der Begriff «Ausgaben» und nicht «Kosten» verwendet, da im Gesundheitswesen fast nur Ausgaben erhoben werden: Ausgaben der öffentlichen Hand, die die Spitäler subventioniert; Ausgaben der Krankenversicherungen, die erbrachte Leistungen vergüten; Ausgaben individueller Haushalte, die Direktzahlungen tätigen und Krankenkassenprämien zahlen. Die reellen Kosten der medizinischen Versorgung sind kaum belegt.

11 Um eine gewisse Vergleichbarkeit zwischen Zürich und Basel herzustellen, wurden alle Daten über Ausgaben, mit Ausnahme der einzelnen Spitäler, auch für den Kanton Basel-Landschaft erhoben. So konnte der Ausgabenanstieg im Kanton Zürich mit demjenigen in Basel-Stadt und -Landschaft verglichen werden.

12 Bundesamt für Statistik, Neuchâtel 2000, 14 Gesundheit, Kosten des Gesundheitswesens, Detaillierte Ergebnisse 1998 und Entwicklung seit 1960, T17 Seite 52.

13 Eidgenössische Finanzverwaltung, Bern 2000, Finanzstatistik der öffentlichen Haushalte, Kantonsfinanzen 1999.

14 KSK/CAMS Konkordat der Schweizerischen Krankenversicherer, Behandlungsfallstatistik z.B. 1996 .
15 Bundesamt für Sozialversicherung 1997; Materialienbericht; Struktur, Verfahren und Kosten der HMO-Praxen; Neue Formen der Krankenversicherung; Untersuchungsbericht 4; Forschungsbericht Nr. 5/98.

16 Basel-Stadt: Kantonsspital (KBS) und das Gemeindespital Riehen; Kanton Zürich: Universitätsspital (USZ), Kantonsspital Winterthur, Stadtspital Waid sowie die Regionalspitäler Wetzikon und Limmattal und das Bezirksspital Affoltern.

17 Bundesamt für Statistik, Jahresdurchschnitt des Landesindexes der Konsumentenpreise (90/91: 5,9\%, 91/92: 4\%, 92/93: 3,3\%, 93/94: 0,9\%, 94/95: 1,8\%, 95/96: 0,8\%, 96/97: 0,5\%, 97/98: 0\%, 98/99: 0,8\%).

18 Lamneck S. Qualitative Sozialforschung. Methoden und Techniken. Bd. 2. München, Weinheim: Psychologie Verlags Union; 1995.

19 Eine detaillierte Analyse der Ausgaben für einzelne Leistungen im ambulanten Bereich ist erst ab 1996, seit es die Versichertenstatistiken der santésuisse gibt, auf Kantonsniveau möglich geworden.

20 Das Spital Wetzikon verzeichnet im Vergleich zu den anderen Spitälern eine enorme Zunahme des Betriebsaufwandes. Laut Angaben des Verwaltungsdirektors dieses Spitals steht dies im Zusammenhang mit Spitalschliessungen (Rüti, Pfäffikon, Bauma, Wald), wodurch sich das Einzugsgebiet des Spitals Wetzikon vergrösserte.

21 Als medizinischen Bedarf ausgewiesen werden Medikamente, Implantate sowie übriges Verbrauchsmaterial.

22 Im Kanton Basel-Stadt erklärt der schrittweise Abbau der Kantonssubventionen an die ÖKK zur Abgeltung der überproportionalen Alterslast eine zusätzliche Erhöhung des durchschnittlichen Prämienniveaus. Die ÖKK muss aufgrund der geringeren Kantonssubventionen die fehlenden Einnahmen aus erhöhten Prämienbeiträgen decken. Dies erlaubt den anderen Krankenkassen, die Prämien ebenfalls vermehrt zu erhöhen und so den Nachholbedarf abzubauen. Denn aufgrund der Konkurrenz zur ÖKK waren diese gezwungen, ihre Beiträge im Kanton Basel-Stadt künstlich tief zu halten und eine Quersubventionierung aus anderen Kantonsbeiträgen zu betreiben.

23 Bundesamt für Sozialversicherung, Sektion Statistik 3003 Bern, Statistik der Krankenversicherung 1998, Bern 12.99, Tabelle E.05.

24 Coulter A, Ham C (eds.). The global challenge of health care rationing. State of Health Series. Philadelphia: Open University press; 2000.

25 Schopper D, Baumann-Hölzle R, Tanner M. Rationierung im Gesundheitswesen: Was könnte die Schweiz von anderen Ländern lernen? Schweiz Ärztezeitung 2002;83:2356-63. 